\section{§29. 2D MHD Simulation of a FRC Plasma with Axial Neutral Gas Flow Injection}

Takahashi, T., Koike, S. (Gunma Univ.), Mizuguchi, N.

Field-Reversed Configurations (FRCs) are highly elongated high-beta plasma. Since they are topologically in a simply-connected shape, therefore they can be translated along the guide field. ${ }^{1)}$ In FRC experiment history, translation technique has been well developed, because the FRC plasma has to be translated from the formation region surrounded by such as the quartz tube to the confinement region in a metal chamber.

In the present study, we numerically investigate an FRC translation process into the confinement region where the neutral gas is filled. The relative motion between translated FRC and background neutral gas at room temperature is equivalent to end-on injection of cold neutral flow into a static FRC plasma. In this point of view, we model a translation process as axial neutral flow injection, and we perform the resistive MHD simulation. In order to consider ionization of neutral particles, we solve the time evolution equation with source terms. For example, the equation of continuity is given by

$$
\frac{\partial \rho}{\partial t}=-\nabla \cdot(\rho \mathbf{u})+\rho_{\mathrm{n}} n_{\mathrm{e}}\langle\sigma v\rangle
$$

We carry out the 2D resistive MHD simulation to find dynamic behavior of an FRC plasma into which neutral gas is injected. Since we are interested in the relation between the effects of the neutral gas flow injection on the flux lifetime of FRC and the injection speed of neutral gas, the simulation is performed for various injection speeds (100, $170,200$, and $250[\mathrm{~km} / \mathrm{s}])$.

The FRC plasma suffers from a resistive decay, and we find the reduction in both the length and radius of the separatrix. In order to observe the decay process, the time evolution of the separatrix radius on the midplane is shown in Fig. 1 where the results for the gas flow speed of 100, 170, 200 , and $250[\mu \mathrm{s}]$ are compared with that without neutral gas flow. Although the flux lifetime increment is small, it is found that the higher injection speed prolongs the FRC lifetime.

Extension of the flux lifetime can be discussed by the Faraday's law. Assuming axisymmetry of the FRC plasma, the surface integral of the Faraday's law on the poloidal cross-section becomes

$$
\frac{\partial \psi}{\partial t}=r\left(u_{z} B_{r}-u_{r} B_{z}\right)-\eta r\left(\frac{\partial B_{r}}{\partial z}-\frac{\partial B_{z}}{\partial r}\right)
$$

in our MHD model. The first term of the R.H.S of Eq. (2) is due to the Lorentz force and the second one is attributed to

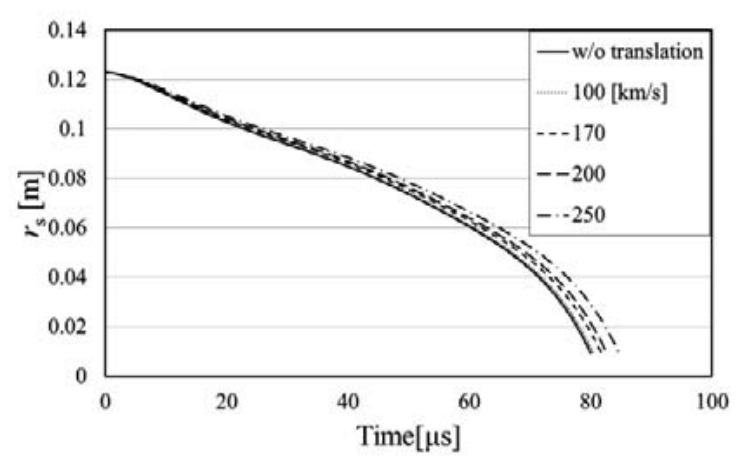

Fig. 1. Time evolution of the separatrix radius dependent on the gas flow velocity

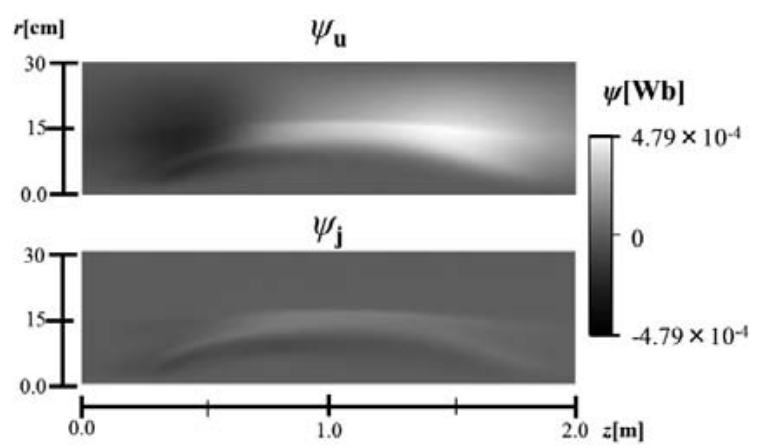

Fig. 2. 2D profile of increment of magnetic flux. Due to (Top) the Lorentz force and (Bottom) the resistive force

the resistive force. To study effects of neutral particle flow, we compare the flux variations for several injection speeds with the case of no neutral flow. In Fig. 2, we present difference in the variations at 43.5 [ $\mu \mathrm{s}]$ (10 Alfvén time) between the injection speed of $250[\mathrm{~km} / \mathrm{s}]$ and no neutral flow case. Here, the neutral particle flows from the left of Fig. 2. Contrary to the gray-colored region, the variation is relatively larger in the white region. It is found from Fig. 2 that influence of the Lorentz force term is larger than the resistive force term. We also notice that the flux increment due to the Lorentz force is significant in the periphery and right of the separatrix. This is partially due to the axial-shift motion (rightward in Fig. 2) of the FRC plasma caused by the neutral particle flow; the interaction between the plasma flow and the external field generates the inductive electric field in the azimuthal direction. We found the peak values of the radial flow increase as the neutral flow speed increases. Therefore, we conclude that the flux lifetime is prolonged by enhancement of the flow across the separatrix; it induced the azimuthal electric field from the Lorentz force. So far, there is no hard evidence of the fact that the increment rate of the flux lifetime depends on the cube of injection speed; it remains as the future subject.

1) Rej, D. J. et al. : Phys. Fluids 29(1986) 852. 